\title{
Reply on "Characteristics and Survival of Korean Anal Cancer From the Korea Central Cancer Registry Data"
}

\author{
Hyoung-Chul Park, Bong Hwa Lee \\ Korea Colorectal Cancer Study Group (KOCCS), Seoul, Korea
}

To the editor:

Thank you for your kind interest. The Korea Central Cancer Registry (KCCR) reports the cancer characteristics for patients in Korea, but this analysis was restricted to the eight most-frequent cancers, such as stomach, colorectal, liver, lung, etc. cancers [1]. Thus, we used the KCCR to focus on uncommon cancers. As you mentioned anal canal cancer tends to present in the sixth and the seventh decades of life, and the median age at diagnosis was 65.2 years in our study population. There was no difference in mean age between males and females in the study.

We reported a relatively lower proportion of squamous cell carcinomas among all anal cancers, which may be one of characteristics of anal cancer in Koreans that is different [2]. Additionally, an adenocarcinoma may include primary a low rectal cancer involving the anal canal [3]. We did not provide full information about the distribution or comparison among the different histologic types or stages. However, analysis of a small number of stratified patients, such as in our study, may lead to bias. As the data are collected, a detailed analysis can be addressed. Unfortunately, as you also pointed out, we did not obtain important data, such as treatment modalities. Chemo-radiation or surgery may be a treatment option in most patients, and both may be needed in some cases [4]. We have a plan to take more clinical information in the future and hope to be able to provide a more valuable report for anal cancer.

I appreciate your additional table including a comparison with other reports. I add that the overall survival has improved with time.

\section{REFERENCES}

1. Jung KW, Won YJ, Kong HJ, Oh CM, Shin A, Lee JS. Survival of Korean adult cancer patients by stage at diagnosis, 2006-2010: national cancer registry study. Cancer Res Treat 2013;45:162-71.

2. Park HC, Jung KW, Kim BW, Shin A, Won YJ, Oh JH, et al. Characteristics and survival of Korean anal cancer from the Korea central cancer registry data. Ann Coloproctol 2013;29:182-5.

3. Habr-Gama A, Sabbaga J, Gama-Rodrigues J, Sao Juliao GP, Proscurshim I, Bailao Aguilar P, et al. Watch and wait approach following extended neoadjuvant chemoradiation for distal rectal cancer: are we getting closer to anal cancer management? Dis Colon Rectum 2013;56:1109-17.

4. Smigielski J, Rychter A, Fijuth J, Brocki M. Advanced anal squamous cell carcinoma: radiotherapy or surgery? Chirurgia (Bucur) 2012;107:626-30. 